\title{
The Risk Assessment and Prediction Tool (RAPT) after Hip and Knee Replacement: A Systematic Review
}

\author{
Cristiano Sconza ${ }^{1}$ Stefano Respizzi ${ }^{1}$ Guido Grappiolo ${ }^{2}$ Marco Monticone ${ }^{3}$
}

${ }^{1}$ Department of Physical and Rehabilitation Medicine, Humanitas Clinical and Research Centre IRCSS, Rozzano, Milan, Italy

2 Hip Diseases and Joint Replacement Surgery Unit, Humanitas Clinical and Research Centre IRCSS, Rozzano, Milan, Italy

${ }^{3}$ Department of Medical Sciences and Public Health, University of Cagliari, Cagliari, Italy

Joints 2019;7:41-45.

\begin{abstract}
Address for correspondence Cristiano Sconza, MD, Department of Physical and Rehabilitation Medicine, Humanitas Clinical and Research Centre IRCSS, Via Alessandro Manzoni 56, 20089, Rozzano, Milan, Italy (e-mail: cristiano.sconza@humanitas.it).
\end{abstract}

\begin{abstract}
Keywords

- Risk Assessment and Prediction Tool

- joint replacement

- joint arthroplasty

Purpose The Risk Assessment and Prediction Tool (RAPT) is an interesting instrument for predicting the discharge destination and length of stay (LOS) for patients after hip or knee arthroplasty. The aim of this review is to describe its predictive ability, current utilization, and future prospects through the analysis of scientific literature.

Methods The databases of PubMed, Web of Sciences, Cochrane Library, and Pedro were searched for English studies on RAPT prediction capacity. Only original prospective or retrospective articles that analyze specifically the use of RAPT were included, whereas those concerned with other preoperative prediction tools or those only considering other aspects of recovery after joint replacements were excluded.

Results A total of 27 references were retrieved, and 8 studies were selected. All analyzed studies demonstrated that RAPT could reduce LOS and accurately predict discharge disposition especially for high- and low-risk patients. In the intermediate risk category, a targeted intensive postoperative rehabilitation program has demonstrated good results in reducing the uncertain outcome.

Conclusion Although contrarily to many of the other scores, the RAPT has been validated in multiple countries with relatively similar results between different institutions; however, its validity has yet to be tested and adapted in every nation context. Further studies confirming the predictive accuracy of RAPT at other institutions are needed as well as studies assessing the effect of using RAPT to identify patients for targeted interventions in terms of LOS, discharge disposition, clinical outcomes, and financial impact.

Level of Evidence This is a level IV, systematic review of level III and IV study.
\end{abstract}

\section{Introduction}

Total joint arthroplasties (TJAs) are some of the most commonly performed elective orthopedic procedures for the management of osteoarthritis $(\mathrm{OA})$. In recent years, the demand for total knee arthroplasty (TKA) and total hip arthroplasty (THA) is growing rapidly. ${ }^{1}$ Although these procedures are safe treatments for $\mathrm{OA}$,

received

December 13, 2017

accepted after revision

June 10, 2019

published online

July 25, 2019
DOI https://doi.org/

10.1055/s-0039-1693459. ISSN 2282-4324. the costs associated with them are substantial. ${ }^{2}$ The socioeconomic burden of care for postoperative rehabilitation of TJA is an emerging widespread issue. The adoption of proper measures is required to correctly allocate resources with respect to the patient needs, avoiding disparities, and containing the costs sustained by the health care system. ${ }^{3}$ Regarding this
Copyright (c) 2019 Georg Thieme Verlag License terms KG Stuttgart · New York 
perspective, in the last years, the attention has been focalized on patients' postoperative management, especially on the inpatient length of stay (LOS) after surgery, encouraging early discharge from hospitals and substitution with home-based alternatives whenever appropriate. ${ }^{4-6}$ The identification of some preoperative objective criteria that would help clinicians to predict patient outcome and needs before surgery became crucial to allow for a more efficient postoperative care management and represent the first step in developing a clinical prediction tool to identify patients at risk for delayed postoperative recovery. ${ }^{7}$ Based on these considerations, in 2003 a method able to identify the risk of needing extended inpatient rehabilitation after arthroplasty was first published. ${ }^{8}$ This 6-item tool was named Risk Assessment and Prediction Tool (RAPT) and generates a score from 1 to 12 with a lower score indicative of higher risks for needing inpatient rehabilitation after joint arthroplasty. In particular, patients with scores $<6$ and $>9$ result in a high and low risk, respectively, whereas patients with scores between 6 and 9 are considered to have an intermediate risk. Initially, the tool contained nine factors. Two of them were excluded since they were not significant: preoperative medical comorbidities and home environment. The factor "patient expectation" was also excluded since it could change according to the patients' education level and it could be modified with preoperative counseling, even though it was by far the most relevant. The final scoring system was composed of the following factors: age, gender, preoperative walking distance, use of gait aids, community support, and presence of a caregiver upon returning home ${ }^{8}$ (-Table 1).

The aim of this systematic review was to describe its predictive utility, current utilization, and future prospects of RAPT after TJA through the analysis of current scientific literature.

\section{Materials and Methods}

\section{Search Strategy}

A computerized search was conducted for English articles published before August 2017. The electronic databases PubMed, Web of Sciences, Cochrane Library, and Pedro were investigated using combination of the following keywords: "RAPT," "RAPT score," "risk assessment and prediction tool," "joint replacement," "hip replacement," "knee replacement," "joint arthroplasty," "hip arthroplasty," "knee arthroplasty," "TJA," “TKA," “TKA," "hip prosthesis," and "knee prosthesis." Database searching was supplemented by screening reference lists and citation tracking included in trials to identify additional studies.

\section{Study Selection Criteria}

Only studies that analyze specifically the use of RAPT were considered, whereas those concerned with other preoperative prediction tools or those only considering other aspects of recovery after joint replacements were excluded.

Original scientific prospective or retrospective study articles were included. Book chapters, expert opinions, reviews, and abstracts of meetings or scientific conference were excluded.

All studied included in the review necessarily had to report at least a measure evaluating our primary endpoint (RAPT prediction capacity).

\section{Results}

\section{Search Strategy Results}

The search strategy identified 27 articles, 15 of which were excluded because they were irrelevant and other 4 were excluded because they were not original articles (precisely 2

Table 1 The Risk Assessment and Prediction Tool (RAPT)

\begin{tabular}{|c|c|c|}
\hline Item & Value & Score \\
\hline \multirow[t]{3}{*}{ 1. What is your age group? } & $50-65$ years & 2 \\
\hline & $65-75$ years & 1 \\
\hline & $>75$ years & 0 \\
\hline \multirow[t]{2}{*}{ 2. Gender? } & Male & 2 \\
\hline & Female & 1 \\
\hline \multirow[t]{3}{*}{ 3. How far, on average, can you walk? (a block is $200 \mathrm{~m}$ ) } & 2 blocks or more ( \pm rests $)$ & 2 \\
\hline & 1-2 blocks (the shopping center) & 1 \\
\hline & Housebound (most of the time) & 0 \\
\hline \multirow[t]{3}{*}{ 4. Which gait aid do you use? (more often than not) } & None & 2 \\
\hline & Single point stick & 1 \\
\hline & Crutches/frame & 0 \\
\hline \multirow{2}{*}{$\begin{array}{l}\text { 5. Do you use community supports? } \\
\text { (home help, meals-on wheels, district nurse) }\end{array}$} & None or one per week & 1 \\
\hline & Two or more per week & 0 \\
\hline \multirow{3}{*}{$\begin{array}{l}\text { 6. Will you live with someone who can care for you after } \\
\text { your operation? }\end{array}$} & Yes & 3 \\
\hline & No & 0 \\
\hline & Your score & $\ldots . . / 12$ \\
\hline
\end{tabular}




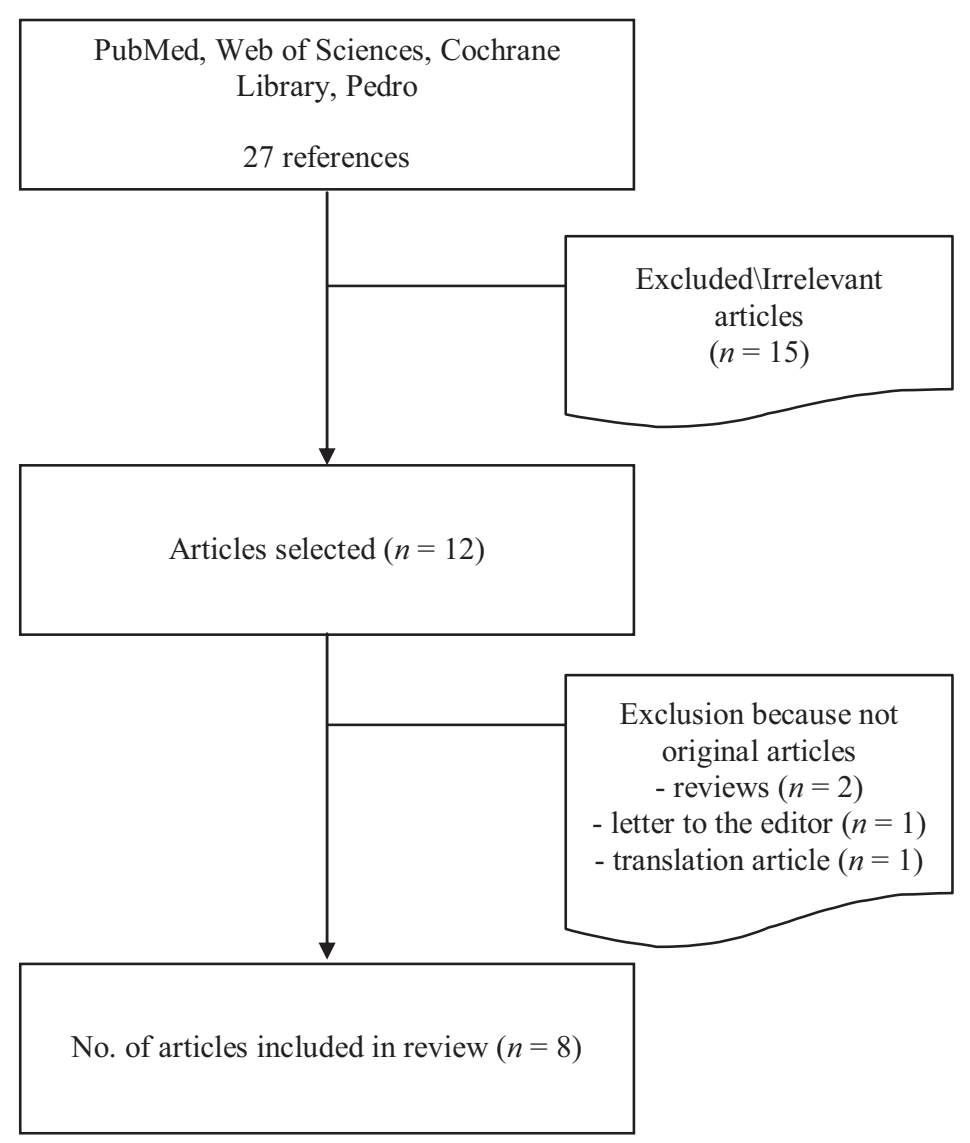

Fig. 1 Flowchart on the search and selection process.

reviews, 1 letter to the editor, and 1 translation and cultural adaptation study).

Applying these criteria led to the inclusion of a total of 8 papers $^{2,4,7-12}$ (-Fig. 1).

\section{Summary of Evidence}

In 2003, the RAPT was developed and validated on data from an Australian cohort of 650 subjects undergoing hip or knee primary arthroplasty or revision procedure. The cohort was split into two groups; data from the first 520 subjects were used to develop the tool and data from the next 130 subjects were used for validation. ${ }^{8}$ Discharge destination was correctly predicted in approximately $75 \%$ of cases, in particular, in patients at highest risk for the need of extended rehabilitation, the accuracy is $89 \% .^{8}$ Between the three classes of risk, the majority of incorrect RAPT predictions occurred within the group scoring 6 to 9 (correct accuracy of $62.3 \%$ ) that identified patients with a less certain and modifiable outcome. The same authors published another article in 2004 which focused on two cohorts of 50 patients who had total knee replacement (TKR) or total hip replacement (THR) surgery. ${ }^{6}$ They investigated whether providing additional postoperative physiotherapy (ranging from 5 to 12 sessions per week) for patients with RAPT scores of 6 to 9 could increase the rate of direct home discharge, thus reducing LOS. The results showed that the percentage of patients directly discharged increased significantly, from 34 to $64 \%$ with no increase in hospital readmission rate. A study from 2011 demonstrated that RAPT scores are correlated with the risk of postoperative complications in patients after TKR. ${ }^{9}$ Subjects with a RAPT score of more than 9 had a low risk of complications, whereas patients with a RAPT score less than 6 had a high risk, confirming that hospitalization is justified for patients in this category. In 2013, Tan et al used RAPT for predicting the destination discharge for a group of 569 patients undergoing TKR. ${ }^{10}$ They found an overall predictive accuracy of $85 \%$ and that RAPT items and scores, particularly the presence of a caregiver at home and preferred discharge destination, can significantly predict the actual discharge destination and LOS. These results were also found in a French study on 134 patients after THA, ${ }^{11}$ that confirmed the usefulness of RAPT in postoperative orientation and that old age, living alone, and patient's preference were determining factors for discharge management. In a study from 2015, conducted on 3,213 American subjects, RAPT accurately predicted discharge disposition for high- and low-risk subjects. ${ }^{2}$ The overall predictive accuracy was $78 \%$, with 80 and $77 \%$ for patients undergoing THA and TKA, respectively. RAPT scores $<6$ and $>10$ predicted discharge to inpatient rehabilitation and home with more than $90 \%$ accuracy. Predictive accuracy was lowest for scores between 7 and 10 at $65.2 \%$ with almost $50 \%$ of patients receiving scores in this range. On the basis of these results, the authors suggested to modify the RAPT risk categories in their population into high risk $<7$, intermediate risk 7 to 10 , and low risk $>10$, as well as to implement targeted interventions to assist discharges for subjects at intermediate risk. To study the relation between RAPT scores and functional 
discharge criteria, Oosting et $\mathrm{l}^{12}$ found that two performancebased tests, the Timed Up and Go test (TUG) ${ }^{13}$ and $10-\mathrm{m}$ walking test $(10 \mathrm{MW}),{ }^{14}$ in association with a more conventional screening concerning patient's age and comorbidities, could give a significant added value in predicting patient's functional recovery after THR. Recently, the RAPT was applied to 535 American subjects undergoing TJA, confirming its properties. ${ }^{7}$ The study was also interesting because, for the first time, RAPT was evaluated in a bundled payment program for TJA, spine fusion, and cardiac valve surgery. At the end of the study, the RAPT has shown the ability to predict discharge disposition for TJR and spine surgery patients, but not cardiac valve surgery patients.

\section{Discussion}

The RAPT was created to be a valid instrument for predicting the discharge destination for patients after an elective hip or knee arthroplasty. During its development this tool has shown significant benefits as well as some limitations. The really interesting aspect is represented not only in its capacity to influence LOS of patients but more importantly in its ability to stratify patients' postoperative risks to individualize the appropriate rehabilitation program and setting according to actual patient necessity while reducing health care costs. All analyzed studies demonstrated that RAPT could reduce LOS and accurately predict discharge disposition especially for high- and low-risk patients while some doubts still remains for those in the intermediate risk category as well as for the range that should be used to classify this risk category, 6 to 9 or 7 to 10 . To reduce the uncertain outcome associated with increasing home discharged of medium risk patients, a targeted intensive postoperative rehabilitation program has demonstrated good results. ${ }^{4}$ Some authors advocate for the use and the identification of other instruments in addition to RAPT for improving predictive accuracy such as some performancebased functional tests like TUG and 10MW or health status scores like the American Society of Anesthesiologists score, ${ }^{15}$ the Charnley classification, ${ }^{16}$ or the body mass index. ${ }^{17}$ However, since one of the best advantages of RAPT is its easy use and linear classification, the introduction of other tools or factors could create many variables resulting in a more complicated instrument that we do not know how to integrate with RAPT. For example, some studies ${ }^{8,11}$ considering the role of comorbidity in discharge outcome, suggested that patients with medical comorbidities, if acceptable candidates for TJA, do not have an increased need for rehabilitation and that the medical criteria seem to have very little influence on postoperative management. This particular aspect could be justified by the long duration of hospitalization that patients had at the time of these studies and the differences existing between health care systems, but remains an interesting field for researchers. In every study, it emerged that patient's preferred discharge destination had a significant influence on LOS and discharge setting, even Oldmeadow el al found that this variable had the highest weighted impact on outcomes ${ }^{8}$ and, to avoid bias, it was taken out of RAPT scoring. But this aspect highlights how patients who might feel confused due to the lack of information on operation and discharge procedures are more afraid to return home and could strongly influence postoperative management. ${ }^{18}$ Therefore, counseling and educating patients and their families before surgery is a fundamental action that, in association with the use of RAPT, could increase patients' readiness and self-confidence for discharge as well as rehabilitate psychological aspects. ${ }^{10}$ Although the RAPT demonstrates unquestionable advantages and potential, some critical points are still present and represent the future directions of research. The clinical and institutional heterogeneity existing between nations and also between hospitals of the same country makes data difficult to extend to other medical institutions. For example, Dauty et al concluded that it would be relevant to conduct a French prospective multicenter study to test specifically in his country the use of the RAPT to orientate patients before TKR surgery. ${ }^{9}$ Hansen et al specified different cutoffs between risk categories, which they speculate may reflect differences between U.S. and Australian patient population and health care systems. ${ }^{2}$ The RAPT was also evaluated in a study from Singapore, in which the authors decided to modify questions 3 and 4 of the questionnaire to be more applicable to the local context. ${ }^{10}$ These aspects underline the necessity to develop a transcultural model and validation in other countries as Coudeyre et al made in France. ${ }^{11,19}$ Although contrarily to many of the other scores, the RAPT has been validated in multiple countries and continents with relatively similar results between different institutions ${ }^{2}$ however, its validity has yet to be tested in every nation context. For this reason, we are already working on cross-cultural adaptation in Italy and to fully validate the RAPT in subjects after TJA in our country. Other limits of current studies concern the differences in patients discharge criteria conditioning the LOS variability, the role of insurance coverage which depends on the health care system of each country, and the postacute rehabilitation care settings.

\section{Conclusion}

The growing demand for TJA and the rising health care costs highlight the need to plan the hospital discharge more efficiently and appropriately. This emphasizes the importance to create specific care pathways based on the preoperative stratification and prediction of patient outcomes and needs to limit the LOS and correctly manage destination discharge. The RAPT has demonstrated good predictive accuracy assisting clinicians with identification of patients for targeted interventions to facilitate home discharge. It is an easy-to-use instrument that has been validated in many countries with relatively similar results. Further studies confirming the predictive accuracy of RAPT at other institutions are needed as well as studies assessing the effect of using RAPT to identify patients for targeted interventions in terms of LOS, discharge disposition, clinical outcomes, and financial impact.

\section{Conflict of Interest}

None declared. 


\section{Acknowledgment}

The authors thank Dr. D.A. Shiffer for helping with the redaction of this paper.

\section{References}

1 Konopka JF, Hansen VJ, Rubash HE, Freiberg AA. Risk assessment tools used to predict outcomes of total hip and total knee arthroplasty. Orthop Clin North Am 2015;46(03):351-362

2 Hansen VJ, Gromov K, Lebrun LM, Rubash HE, Malchau H, Freiberg AA. Does the Risk Assessment and Prediction Tool predict discharge disposition after joint replacement? Clin Orthop Relat Res 2015;473 (02):597-601

3 Benedetti MG, Sarti D, Bonfiglioli Stagni S, Marian E. Setting, clinical pathways, fast-track and rehabilitation following primary knee arthroplasty: a literature review. Open Rehabil J 2015;8:17-24

4 Oldmeadow LB, McBurney H, Robertson VJ, Kimmel L, Elliott B. Targeted postoperative care improves discharge outcome after hip or knee arthroplasty. Arch Phys Med Rehabil 2004;85(09): 1424-1427

5 Monticone M, Ferrante S, Rocca B, et al. Home-based functional exercises aimed at managing kinesiophobia contribute to improving disability and quality of life of patients undergoing total knee arthroplasty: a randomized controlled trial. Arch Phys Med Rehabil 2013;94(02):231-239

6 Monticone M, Ambrosini E, Rocca B, Lorenzon C, Ferrante S, Zatti G. Task-oriented exercises and early full weight-bearing contribute to improving disability after total hip replacement: a randomized controlled trial. Clin Rehabil 2014;28(07):658-668

7 Slover J, Mullaly K, Karia R, et al. The use of the Risk Assessment and Prediction Tool in surgical patients in a bundled payment program. Int J Surg 2017;38:119-122

8 Oldmeadow LB, McBurney H, Robertson VJ. Predicting risk of extended inpatient rehabilitation after hip or knee arthroplasty. J Arthroplasty 2003;18(06):775-779
9 Dauty M, Schmitt X, Menu P, Rousseau B, Dubois C. Using the Risk Assessment and Predictor Tool (RAPT) for patients after total knee replacement surgery. Ann Phys Rehabil Med 2012;55(01):4-15

10 Tan C, Loo G, Pua YH, et al. Predicting discharge outcomes after total knee replacement using the Risk Assessment and Predictor Tool. Physiotherapy 2014;100(02):176-181

11 Coudeyre E, Eschalier B, Descamps S, et al. Transcultural validation of the Risk Assessment and Predictor Tool (RAPT) to predict discharge outcomes after total hip replacement. Ann Phys Rehabil Med 2014;57(03):169-184

12 Oosting E, Hoogeboom TJ, Appelman-de Vries SA, Swets A, Dronkers JJ, van Meeteren NL. Preoperative prediction of inpatient recovery of function after total hip arthroplasty using performance-based tests: a prospective cohort study. Disabil Rehabil 2016;38(13):1243-1249

13 Mathias S, Nayak US, Isaacs B. Balance in elderly patients: the "getup and go" test. Arch Phys Med Rehabil 1986;67(06):387-389

14 Peters DM, Fritz SL, Krotish DE. Assessing the reliability and validity of a shorter walk test compared with the 10-Meter Walk Test for measurements of gait speed in healthy, older adults. J Geriatr Phys Ther 2013;36(01):24-30

15 Wolters U, Wolf T, Stützer H, Schröder T. ASA classification and perioperative variables as predictors of postoperative outcome. $\mathrm{Br}$ J Anaesth 1996;77(02):217-222

16 Charnley J. The long-term results of low-friction arthroplasty of the hip performed as a primary intervention. J Bone Joint Surg Br 1972;54(01):61-76

17 Keys A, Fidanza F, Karvonen MJ, Kimura N, Taylor HL. Indices of relative weight and obesity. J Chronic Dis 1972;25(06):329-343

18 Perry MA, Hudson S, Ardis K. "If I didn't have anybody, what would I have done?": Experiences of older adults and their discharge home after lower limb orthopaedic surgery J Rehabil Med 2011; 43(10):916-922

19 Coudeyre E, Descamps S, Intyre JM, Boisgard S, Poiraudeau S, Lefevre-Colau MM. Translation and French cultural adaptation of a decision making tool for patients orientation after total hip or knee arthroplasty. Ann Phys Rehabil Med 2009;52(10):694-703 